\title{
Local CuO Nanowire Growth on Microhotplates: In Situ Electrical Measurements and Gas Sensing Application
}

\author{
Stephan Steinhauer ${ }^{\dagger}$, Audrey Chapelle ${ }^{\ddagger}$, Philippe Menini ${ }^{\ddagger}$, Mukhles Sowwan*,† \\ ${ }^{\dagger}$ Nanoparticles by Design Unit, Okinawa Institute of Science and Technology (OIST) Graduate University, \\ 1919-1 Tancha Onna-Son, Okinawa 904-0495, Japan

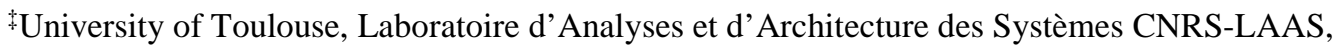 \\ 7 Avenue du Colonel Roche, 31031 Toulouse Cedex 4, France \\ KEYWORDS: CuO nanowire, gas sensor, microhotplate, in situ measurement, CO sensing, sensor degradation
}

\begin{abstract}
We report on local CuO nanowire growth on microhotplates combined with in situ measurement of the electrical resistance for well-controlled integration into conductometric gas sensing devices. Discrete current steps were observed during the CuO nanowire synthesis process, which is attributed to individual nanowire connections being formed. The high gas sensitivity of the CuO nanowire devices was confirmed by detection of carbon monoxide $\mathrm{CO}$ in the low ppm-level concentration range. Furthermore, we demonstrate that CuO nanowire growth inside a gas measurement setup allows studies on gas sensor poisoning/deactivation processes. A significant decrease of CO response was found after controlled exposure to humidity, which suggests sensor deactivation by surface hydroxylation. Thus our approach could be a novel and simple way for revealing new insights in various gas sensor degradation mechanisms in the future and might be also adapted for different metal oxide nanomaterials.
\end{abstract}

Semiconducting nanowires are considered as promising candidates for the realization of next-generation electronic devices ranging from transistors, memories and optoelectronic systems to chemical/biological sensing ${ }^{1,2}$. Conductometric gas sensors rely on electrical resistance changes due to reactions with surrounding gas molecules, which enables potential applications such as toxic gas alarms or atmospheric pollution monitoring ${ }^{3}$. Semiconducting metal oxide nanowires were found to be suitable for highly sensitive detection of different target gases and have been thoroughly studied in various device configurations ${ }^{4,5}$.

Cupric oxide $(\mathrm{CuO})$ nanowires have received growing attention due to high sensitivity for toxic gases such as carbon monoxide $\mathrm{CO}^{6,7,8}$, nitrogen dioxide $\mathrm{NO}_{2}{ }^{6,9}$ or hydrogen sulfide $\mathrm{H}_{2} \mathrm{~S}^{7,10,11}$. In addition, this material also shows promising properties for a variety of other applications, such as rechargeable battery electrodes ${ }^{12}$, photovoltaics $^{13}$, infrared detectors ${ }^{14}$ and field emitting devices ${ }^{15}$. $\mathrm{CuO}$ nanowires can be grown on a variety of $\mathrm{Cu}$ substrates using a simple, cheap and catalyst-free thermal oxidation method ${ }^{16}$. Most importantly, $\mathrm{CuO}$ nanowire growth was demonstrated on $\mathrm{Cu}$ thin films at temperatures compatible with CMOS back-end technology ${ }^{17}$, which is a crucial prerequisite for potential integration with standard industrial processes. Thermal oxidation synthesis of $\mathrm{CuO}$ nanowires was also achieved by local heating on MEMS-based resistive microheaters ${ }^{18}$ (in the following termed microhotplates) as an alternative to conventional techniques relying on heating the whole sample in a furnace or on a hotplate. Furthermore, $\mathrm{CuO}$ nanowires were successfully integrated on CMOS microhotplates by local growth between adjacent $\mathrm{Cu}$ microstructures leading to functional devices for gas sensing applications ${ }^{19}$. In this letter, we report on local $\mathrm{CuO}$ nanowire growth in controlled gas atmosphere by heating microhotplate devices inside a gas measurement setup, while measuring the electrical resistance of the devices in situ during the nanowire synthesis process. In contrast to common gas sensor fabrication methods, our approach reveals insights into the electrical properties of single $\mathrm{CuO}$ nanowires in an array configuration and enables the characterization of sensing properties immediately after the nanowire synthesis process. As a consequence, this technique is ideally suited for studying sensor poisoning/deactivation processes, which we demonstrate by comparing sensing performance before and after controlled exposure to humidity. We focus on the colorless and odorless toxic gas $\mathrm{CO}$, a common environmental pollutant which is produced from various different sources such as motor vehicles or gas appliances. Adverse human health effects for $\mathrm{CO}$ exposure in the ppm-level concentration range have been reported ${ }^{20}$, which explains the demand for miniaturized, high-performance gas sensing devices for CO detection.

The microhotplate devices ${ }^{21}$ used for this study were based on a multi-layer $\mathrm{SiO}_{2}-\mathrm{SiN}_{\mathrm{x}}$ membrane released by deep reactive ion etching of the Si substrate from the wafer backside. The embedded heater layer consisted of a Ti/Pt thin film and was insulated by a $\mathrm{SiO}_{2}$ layer deposited by plasma-enhanced chemical vapor deposition. Interdigitated $\mathrm{Ti} / \mathrm{Pt}$ electrodes with circular shape were used as sensor contacts. More details on the microhotplate layout and device technology can be found in ref 22. On top of the microhotplate surface, $\mathrm{Cu}$ microstructures (Cu thickness $\sim 600 \mathrm{~nm} ; 15 \mathrm{~nm}$ Ti adhesion layer) were fabricated by thermal evaporation and lift-off, which is shown in Figure 1. The layout consisted of $\mathrm{Cu}$ structures (length of opposing edges $20 \mu \mathrm{m}$ ) placed onto the interdigitated electrodes with a gap distance of around $2 \mu \mathrm{m}$. In the following, these $\mathrm{Cu}$ structures will serve as substrate for the growth of $\mathrm{CuO}$ nanowires, which bridge the gap between the adjacent electrodes, similar as in ref 23. 


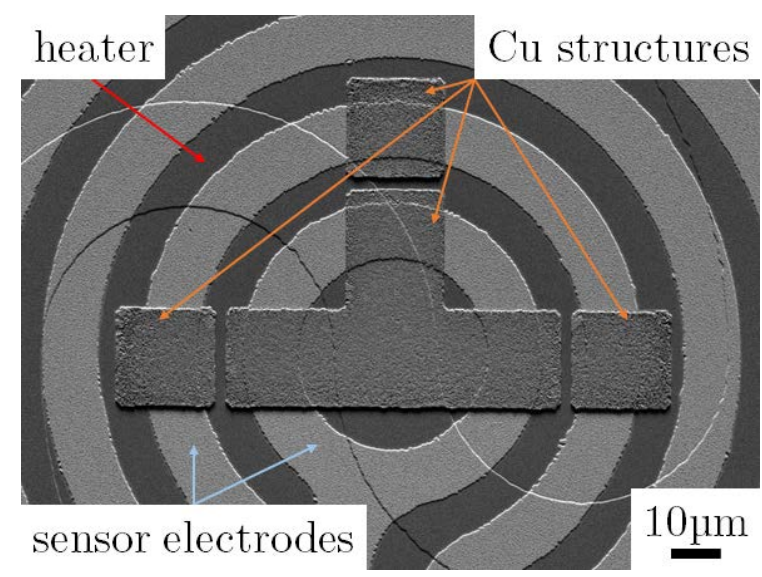

Figure 1. Scanning electron microscopy image of the device design for $\mathrm{CuO}$ nanowire growth. The gap distances between $\mathrm{Cu}$ microstructures connected to different sensor electrodes are around $2 \mu \mathrm{m}$, whereas the lengths of opposing edges are $20 \mu \mathrm{m}$. After thermal oxidation, $\mathrm{CuO}$ nanowires bridged the gap and formed an electrical connection.

The microhotplate devices were glued onto a printed circuit board and connected to Au pads by wire bonding. $\mathrm{CuO}$ nanowire growth and gas sensing measurements were performed inside a test chamber consisting of standard vacuum components. The gas composition was controlled by means of mass flow controllers using a constant total gas flow of 500sccm. A Keithley 2636A dual-channel SourceMeter was employed for microhotplate heating as well as electrical measurements during $\mathrm{CuO}$ nanowire growth and gas sensing experiments.

For $\mathrm{CuO}$ nanowire growth in dry synthetic air, microhotplates were biased with a constant voltage at a power consumption of $39 \mathrm{~mW}$, which corresponds to around $335^{\circ} \mathrm{C}$. The nanowire synthesis conditions are comparable to those in previous studies ${ }^{19,23}$. During the thermal oxidation experiments, a constant voltage was applied while measuring the current between the sensor electrodes. The first $125 \mathrm{~min}$ of a representative $\mathrm{CuO}$ nanowire growth experiment with in situ electrical measurements is shown in Figure 2 $(10 \mathrm{mV}$ bias). The current increases in discrete steps, which is interpreted by single $\mathrm{CuO}$ nanowires bridging the gap between the oxidized $\mathrm{Cu}$ microstructures (see inset of Figure 2 for an SEM image after $\mathrm{CuO}$ nanowire synthesis for $5 \mathrm{~h}$ ). The total electrical resistance after growth may be interpreted by a parallel circuit of multiple $\mathrm{CuO}$ nanowires, whereas the heights of the current steps during the in situ experiments corresponds to the electrical conductance of single nanowires. When assuming cylindrical nanowire geometry and typical dimensions (diameter $20 \mathrm{~nm}$, length $1 \mu \mathrm{m}$ ), the observed conductance steps can be correlated with nanowire electrical conductivity. In our growth experiments at $335^{\circ} \mathrm{C}$ in dry synthetic air, we commonly observed conductance step values of several tens of nS. Considering the geometrical assumptions mentioned above, this corresponds to roughly $10^{2} \mathrm{~S} / \mathrm{m}$. This crude estimation of typical nanowire electrical conductivities leads to values that are reasonably comparable to four point measurements on single $\mathrm{CuO}$ nanowires with considerably larger diameters in similar environmental conditions ${ }^{24}$. Additional transmission electron microscopy results for $\mathrm{CuO}$ nanowire characterization can be found in the supporting information (Figure S1). The material composition was confirmed by comparing the electron energy loss nearedge fine structure with literature results for $\mathrm{CuO}$. Furthermore, bicrystalline nanowire structure was typically observed, which is a well-known characteristic of $\mathrm{CuO}$ nanowires grown by the thermal oxidation method ${ }^{16,17}$.

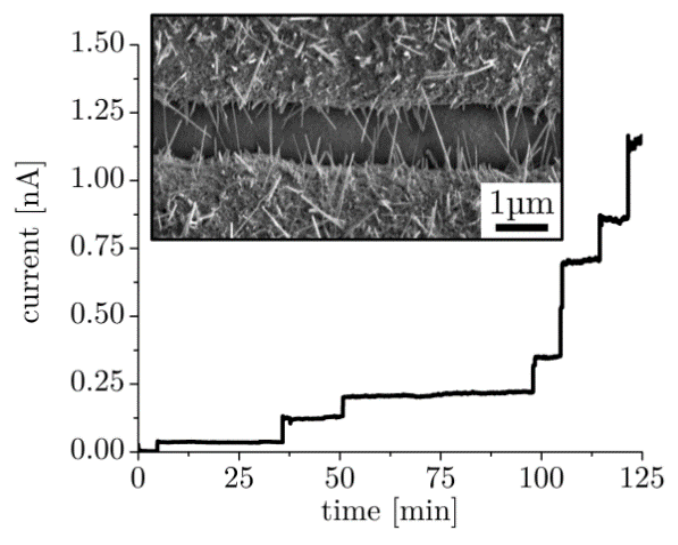

Figure 2. Step-like features of electrical current across sensor electrodes (bias $10 \mathrm{mV})$ during $\mathrm{CuO}$ nanowire growth $\left(335^{\circ} \mathrm{C}\right.$, dry synthetic air). The inset shows a scanning electron microscopy image of $\mathrm{CuO}$ nanowires bridging the gap between oxidized $\mathrm{Cu}$ microstructures after the experiment.

In principle, the presented method enables controlling the number of $\mathrm{CuO}$ nanowire connections during device fabrication. The rise and fall times of microhotplate temperature are in the ms range (20-30ms) and thus the thermal oxidation process may be stopped with high temporal precision. Thus in situ electrical measurements during $\mathrm{CuO}$ nanowire growth might be used for studies on devices with single or few nanowires bridging the gap between the oxidized $\mathrm{Cu}$ structures in the future. Here, we chose a constant time of five hours for $\mathrm{CuO}$ nanowire growth in order to maximize the number of connecting nanowires in the array devices. $\mathrm{CuO}$ nanowire growth rate was previously reported to follow a parabolic law over time and is becoming increasingly low after few hours ${ }^{25}$. Although we assume that in situ electrical measurements might be useful in order to gain insights on $\mathrm{CuO}$ nanowire growth kinetics, we restrict ourselves to the gas sensing application in this letter.

In Figure 3, the $\mathrm{CO}$ sensor response of a typical $\mathrm{CuO}$ nanowire device, which was grown inside the gas measurement setup, is shown. The measurement was performed after sensor stabilization at a temperature of around $325^{\circ} \mathrm{C}$ (microhotplate power consumption $37 \mathrm{~mW}$ ) after a $\mathrm{CuO}$ nanowire synthesis process at around $335^{\circ} \mathrm{C}$ for five hours. The presented $\mathrm{CuO}$ nanowire device showed sensor responses from $6.4 \%$ to $27.6 \%$ for CO concentrations from $1 \mathrm{ppm}$ to 30ppm, respectively. Supplementary data on the CO response can be found in the supporting information (Figure S2). $\mathrm{CuO}$ nanowires are suspended and entirely surrounded by the gas atmosphere, which is a highly favorable configuration. It is known from literature that suspended nanomaterials are advantageous due to faster adsorption/desorption kinetics ${ }^{26}$, maximization of surface area exposed to the gas atmosphere ${ }^{27}$ and improved signal-to-noise ratio ${ }^{28}$. The excellent CO sensing performance is comparable to literature results on $\mathrm{SnO}_{2}$ nanowire array devices ${ }^{29}$ and thick films of $\mathrm{CuO}$ microspheres ${ }^{30}$ in dry air. Increased $\mathrm{CuO}$ nanowire resistance was found, which is in accordance with previous results on similar devices $^{7}$ and with other literature reports ${ }^{6,8,31}$. It can be assumed that the $\mathrm{CO}$ response can be explained by sensing mechanisms of metal oxides proposed in literature, which rely on the oxygen chemisorption model ${ }^{32}$. In the case of the p-type metal oxide $\mathrm{CuO}$, $\mathrm{CO}$ molecules are expected to react with chemisorbed oxygen ions to form $\mathrm{CO}_{2}$, which leads to a reduced hole concentration in the surface accumulation layer and thus an increase in electrical resistance ${ }^{30,31}$. Additional gas measurement results showed increased $\mathrm{CuO}$ nanowire resistance in the presence of $\mathrm{H}_{2} \mathrm{~S}$ and decreased resistance during $\mathrm{NO}_{2}$ exposure (Figure S3), which is in accordance with the oxygen chemisorption model ${ }^{9,11}$. 


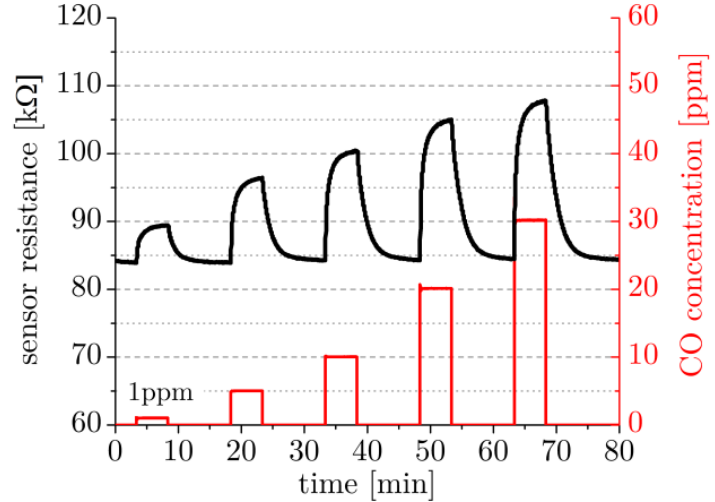

Figure 3. Electrical resistance of $\mathrm{CuO}$ nanowire gas sensor device in dry synthetic air during pulses of carbon monoxide $\mathrm{CO}$ exposure (1-30ppm) at an operation temperature of $325^{\circ} \mathrm{C}$.

$\mathrm{CuO}$ nanowire device integration by growth between adjacent $\mathrm{Cu}$ microstructures does not require any further post processing steps and thus avoids nanowire surface contamination, which is most commonly detrimental to the device performance of sensors and detectors ${ }^{33}$. Furthermore, local $\mathrm{CuO}$ nanowire growth on microhotplates inside a gas measurement setup offers the possibility of performing sensing experiments immediately after nanowire synthesis. The sensors may be characterized without exposure to ambient atmosphere, which usually contains a variety of reactive species, for instance water vapor and volatile organic compounds. As a consequence, this enables gas sensing measurements on devices with pristine $\mathrm{CuO}$ nanowire surfaces, which is an ideal starting point for studies on sensor deactivation mechanisms. Various degradation mechanisms have been reported in literature, such as sensor poisoning by sulfur or carbon compounds and humidity-induced changes ${ }^{34}$. Here, we restrict ourselves to the influence of water vapor as exemplary case.

$\mathrm{CuO}$ nanowire growth inside a gas measurement chamber followed by gas sensing characterization was utilized in order to study the sensor response to $\mathrm{CO}$ before and after controlled exposure to water vapor. This methodology differs from previous literature reports investigating the humidity influence on gas sensing properties of $\mathrm{CuO}$ nanomaterials ${ }^{23,35,36}$, as they compared the gas sensor response in dry and humid air. In contrast, we address irreversible changes of $\mathrm{CuO}$ nanowire gas sensor performance induced by the presence of water vapor. Experiments were performed as follows: $\mathrm{CuO}$ nanowire gas sensors at a microhotplate temperature around $325^{\circ} \mathrm{C}$ were exposed to a $5 \mathrm{~min}$ pulse of humidity by applying a mixed gas flow of 300sccm dry air and 200sccm humid air (through bubble humidifier), which corresponds to around 30\% relative humidity at room temperature. During the humidity pulse, the device resistance increased to more than three times of its value in dry air. The $\mathrm{CuO}$ nanowire gas sensor response to 30ppm CO in dry air was characterized before and after humidity exposure (sensor recovery for 3h), which is shown in Figure 4. As can be seen, the CO sensor response of the $\mathrm{CuO}$ nanowire device considerably decreased after humidity exposure, whereas the sensor resistance baseline was markedly increased. Comparable $\mathrm{CuO}$ nanowire gas sensor deactivation characteristics after humidity exposure were found in multiple experiments. These findings underline the crucial influence of $\mathrm{CuO}$ nanowire device pre-treatment on gas sensor performance for CO detection.

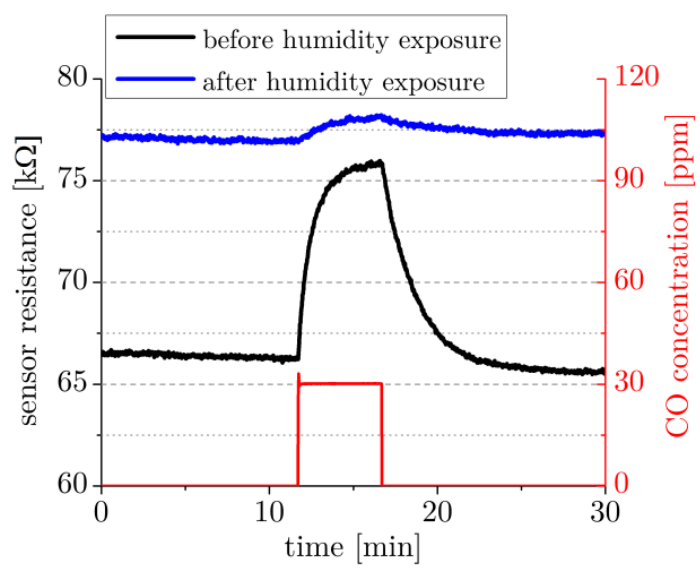

Figure 4. Comparison of $\mathrm{CuO}$ nanowire gas sensor response to 30ppm carbon monoxide CO (dry synthetic air; operation temperature $325^{\circ} \mathrm{C}$ ) before and after humidity exposure.

We assume that the notable decrease of CO sensing response after water vapor exposure may be explained by hydroxylation of the $\mathrm{CuO}$ nanowire surfaces. It is expected that hydroxyl groups lead to a decrease of surface sites for oxygen chemisorption, similar as was suggested due to experimental results on $\mathrm{CuO}$ thick films ${ }^{36}$ and theoretical calculations for the case of $\mathrm{SnO}_{2}{ }^{37}$. This argument is consistent with the increased sensor baseline resistance (see Figure 4) after humidity exposure, because a decrease of chemisorbed oxygen ions would result in a lower number of holes in the $\mathrm{CuO}$ surface accumulation layer ${ }^{36}$ leading to decreased surface conductivity. Furthermore, a lower number of chemisorbed oxygen ions is expected to result in a decrease of CO surface reactions, which would explain the lower sensor response. However, a detailed understanding of the underlying mechanisms is lacking at the moment and would require extensive atomistic modelling, which is beyond the scope of this work.

The decreased $\mathrm{CO}$ response of $\mathrm{CuO}$ nanowire sensors after water vapor exposure has to be considered for practical gas sensing applications in ambient air. In order to enhance $\mathrm{CO}$ sensing performance in humid atmosphere, $\mathrm{CuO}$ nanowire devices may be decorated with noble metal nanoparticles for increased catalytic activity. We recently demonstrated that magnetron sputter inert gas aggregation is ideally suited for the deposition of size-selected Pd nanoparticles on $\mathrm{CuO}$ nanowire surfaces, which improved the $\mathrm{CO}$ sensor response in humid air considerably ${ }^{8}$. Furthermore, nanoparticle decoration is expected to improve sensor selectivity to other gases such as $\mathrm{H}_{2} \mathrm{~S}$ or $\mathrm{NO}_{2}$, which is a common limitation of metal oxidebased devices ${ }^{3}$. Alternatively, sensor properties might be enhanced by plasma treatments, as the latter were found to enhance the catalytic activity of $\mathrm{CuO}$ nanowires for $\mathrm{CO}$ oxidation ${ }^{38}$.

In summary, we performed local $\mathrm{CuO}$ nanowire growth on microhotplates and observed discrete current steps during simultaneous in situ measurements of the electrical resistance, which is attributed to individual nanowire connections being formed. Our approach offers the possibility of performing gas sensing experiments immediately after nanowire growth on devices with pristine $\mathrm{CuO}$ surfaces. Excellent gas sensor performance for the detection of CO concentrations down to $1 \mathrm{ppm}$ was observed. However, a considerable decrease of CO response was found after exposure to humidity, which is attributed to surface hydroxylation and gives valuable insights in sensor deactivation mechanisms of $\mathrm{CuO}$ nanowire devices. It can be assumed that the presented method might be adapted for other gas-sensitive metal oxide materials, in particular those that allow nanowire synthesis by thermal oxidation at moderate temperatures such as $\mathrm{ZnO}^{39}$ or $\mathrm{Fe}_{2} \mathrm{O}_{3}{ }^{40}$. As a consequence, we expect that metal oxide nanowire growth inside a gas measurement 
setup combined with in situ electrical measurements will lead to new insights on various gas sensor degradation mechanisms in the future.

\section{ASSOCIATED CONTENT}

\section{Supporting Information}

The Supporting Information is available free of charge on the ACS Publications website. $\mathrm{CuO}$ nanowire characterization and gas sensing results (PDF).

\section{AUTHOR INFORMATION}

\author{
Corresponding Author \\ *E-mail: mukhles@oist.jp. \\ Notes \\ The authors declare no competing financial interests.
}

\section{ACKNOWLEDGMENT}

This work was supported by funding from OIST Graduate University. This work was also partly supported by the French RENATECH network through technology developments in the CNRS-LAAS cleanroom.

\section{REFERENCES}

(1) Hayden, O.; Agarwal, R.; Lu, W. Semiconductor nanowire devices. Nano Today 2008, 3, 12-22.

(2) Yang, P.; Yan, R.; Fardy, M. Semiconductor nanowire: what's next? Nano Lett. 2010, 10, 1529-1536.

(3) Kim, I. D.; Rothschild, A.; Tuller, H. L. Advances and new directions in gas-sensing devices. Acta Mater. 2013, 61 974-1000.

(4) Comini, E.; Sberveglieri, G. Metal oxide nanowires as chemical sensors. Mater. Today 2010, 13, 36-44.

(5) Ramgir, N. S.; Yang, Y.; Zacharias, M. Nanowire-based sensors. Small 2010, 6, 1705-1722.

(6) Kim, Y.-S.; Hwang, I.-S.; Kim, S.-J.; Lee, C.-Y.; Lee, J.-H. CuO nanowire gas sensors for air quality control in automotive cabin. . Sens. Actuators B 2008, 135, 298-303.

(7) Steinhauer, S.; Brunet, E.; Maier, T.; Mutinati, G. C.; Köck, A.; Freudenberg, O.; Gspan, C.; Grogger, W.; Neuhold, A.; Resel, R. Gas sensing properties of novel $\mathrm{CuO}$ nanowire devices. Sens. Actuators $B$ 2013, 187, 50-57.

(8) Steinhauer, S.; Singh, V.; Cassidy, C.; Gspan, C.; Grogger, W.; Sowwan, M.; Köck, A. Single CuO nanowires decorated with size-selected Pd nanoparticles for CO sensing in humid atmosphere. Nanotechnology 2015, 26, 175502.

(9) Li, D.; Hu, J.; Wu, R.; Lu, J. G. Conductometric chemical sensor based on individual CuO nanowires. Nanotechnology 2010, 21, 485502.

(10) Chen, J.; Wang, K.; Hartman, L.; Zhou, W. $\mathrm{H}_{2} \mathrm{~S}$ detection by vertically aligned $\mathrm{CuO}$ nanowire array sensors, J. Phys. Chem. C 2008, 112, 16017-16021.

(11) Kim, H.; Jin, C.; Park, S.; Kim, S.; Lee, C. $\mathrm{H}_{2} \mathrm{~S}$ gas sensing properties of bare and Pd-functionalized CuO nanorods. Sens. Actuators B 2012, 161, 594-9.

(12) Yuan, S.; Huang, X.-L.; Ma, D.-L.; Wang, H.-G.; Meng, F.-Z., Zhang, X.-B. Engraving Copper Foil to Give Large-Scale Binder-Free Porous $\mathrm{CuO}$ Arrays for a High-Performance Sodium-Ion Battery Anode. Adv. Mater. 2014, 26, 2273-2279.

(13) Wang, P.; Zhao, X.; Li, B. ZnO-coated CuO nanowire arrays: fabrications, optoelectronic properties, and photovoltaic applications. Opt. Express 2011, 19, 11271-11279.

(14) Wang, S. B.; Hsiao, C. H.; Chang, S. J.; Lam, K. T.; Wen, K. H.; Hung, S. C.; Young, S. J.; Huang, B.R. A CuO nanowire infrared photodetector. Sens. Actuators A 2011, 171, 207-211.

(15) Zhu, Y. W.; Yu, T.; Cheong, F. C.; Xu, X. J.; Lim, C. T.; Tan, V. B. C.; Thong, J. T. L.; Sow, C. H. Large-scale synthesis and field emission properties of vertically oriented $\mathrm{CuO}$ nanowire films. Nanotechnology 2005, 16, 88-92.

(16) Jiang X.; Herricks, T.; Xia, Y. CuO nanowires can be synthesized by heating copper substrates in air. Nano Lett. 2002, 2, 1333-1338.
(17) Zhang, K.; Rossi, C.; Tenailleau, C.; Alphonse, P.; Chane-Ching, J.-Y. Synthesis of large-area and aligned copper oxide nanowires from copper thin film on silicon substrate. Nanotechnology 2007, 18, 275607.

(18) Zhang, K.; Yang, Y.; Pun, E. Y. B.; Shen, R. Local and CMOScompatible synthesis of $\mathrm{CuO}$ nanowires on a suspended microheater on a silicon substrate. Nanotechnology 2010, 21, 235602.

(19) Steinhauer, S.; Maier, T.; Mutinati, G. C.; Rohracher, K.; Siegert, J.; Schrank, F.; Köck, A. Local synthesis of CuO nanowires on CMOS microhotplates for gas sensing applications. in Proceedings of TechConnect World Nanotech, Washington DC, USA; CRC Press: Boca Raton, FL, 2014; pp.53-56.

(20) Omaye, S. T. Metabolic modulation of carbon monoxide toxicity. Toxicology 2002, 180, 139-150.

(21) Ghaddab, B.; Berger, F.; Sanchez, J. B.; Menini, P.; Mavon, C.; Yoboue, P.; Potin, V. Benzene monitoring by micro-machined sensors with $\mathrm{SnO}_{2}$ layer obtained by using micro-droplet deposition technique. Sens. Actuators B 2011, 152, 68-72.

(22) Yoboue, P.; Konate, A.; Asseu, O.; Tety, P.; Menini, P. An efficient nanoparticles-SnO $\mathrm{Sn}_{2}$ gas sensor for industrial applications. Int. J. Mater. Eng. Techn. 2014, 11, 41-56.

(23) Steinhauer, S.; Brunet, E.; Maier, T.; Mutinati, G. C.; Köck, A. Suspended $\mathrm{CuO}$ nanowires for ppb level $\mathrm{H}_{2} \mathrm{~S}$ sensing in dry and humid atmosphere. Sens. Actuators B 2013, 186, 550-556.

(24) Steinhauer, S.; Köck, A.; Gspan, C.; Grogger, W.; Vandamme, L. K. J.; Pogany, D. Low-frequency noise characterization of single $\mathrm{CuO}$ nanowire gas sensor devices. Appl. Phys. Lett. 2015, 107, 123112.

(25) Gonçalves, A. M. B.; Campos, L. C.; Ferlauto, A. S.; Lacerda, R. $\mathrm{G}$. On the growth and electrical characterization of $\mathrm{CuO}$ nanowires by thermal oxidation. J. Appl. Phys. 2009, 106, 034303.

(26) Ahn, M.-W.; Park, K.-S.; Heo, J.-H.; Kim, D.-W.; Choi, K. J.; Park, J.-G. On-chip fabrication of $\mathrm{ZnO}$-nanowire gas sensor with high gas sensitivity. Sens. Actuators B 2009, 138, 168-173.

(27) Yavari, F.; Koratkar, N. Graphene-based chemical sensors. $J$. Phys. Chem. Lett. 2012, 3, 1746-1753.

(28) Chikkadi, K.; Muoth, M.; Liu, W.; Maiwald, V.; Hierold, C. Enhanced signal-to-noise ratio in pristine, suspended carbon nanotube gas sensors. Sens. Actuators B 2014, 196, 682-690.

(29) Sysoev, V. V.; Goschnick, J.; Schneider, T.; Strelcov, E.; Kolmakov, A. A gradient microarray electronic nose based on percolating $\mathrm{SnO}_{2}$ nanowire sensing elements. Nano Lett. 2007, 7, 3182-3188.

(30) Zhang, Y.; He, X.; Li, J.; Zhang, H.; Gao, X. Gas-sensing properties of hollow and hierarchical copper oxide microspheres. Sens. Actuators B 2007, 128, 293-298.

(31) Liao, L.; Zhang, Z.; Yan, B.; Zheng, Z.; Bao, Q. L.; Wu, T.; Li, C. M.; Shen, Z. X.; Zhang, J. X.; Gong, H.; Li, J. C.; Yu, T. Multifunctional $\mathrm{CuO}$ nanowire devices: p-type field effect transistors and $\mathrm{CO}$ gas sensors. Nanotechnology 2009, 20, 085203.

(32) Barsan, N.; Weimar, U. Understanding the fundamental principles of metal oxide based gas sensors; the example of $\mathrm{CO}$ sensing with $\mathrm{SnO}_{2}$ sensors in the presence of humidity. J. Phys.: Condens. Matter 2003, 15, R813-R839.

(33) Li, Y.; Delaunay, J. J. In Nanowires; Prete, P., Ed.; InTech: Rijeka, 2010; pp. 373-394.

(34) Korotcenkov, G.; Cho, B. K. Instability of metal oxide-based conductometric gas sensors and approaches to stability improvement (short survey). Sens. Actuators B 2011, 156, 527-538.

(35) Zhang, F.; Zhu, A.; Luo, Y.; Tian, Y.; Yang, J.; Qin, Y. CuO nanosheets for sensitive and selective determination of $\mathrm{H}_{2} \mathrm{~S}$ with high recovery ability. J. Phys. Chem. C 2010, 114, 19214-19219.

(36) Hübner, M.; Simion, C. E.; Tomescu-Stanoiu, A.; Pokhrel, S.;

Barsan, N.; Weimar, U. Influence of humidity on CO sensing with ptype $\mathrm{CuO}$ thick film gas sensors. Sens. Actuators B 2011, 153, 347-353.

(37) Skouras, E. D.; Burganos, V. N.; Payatakes, A. C. Simulation of gas diffusion and sorption in nanoceramic semiconductors. J. Chem. Phys. 1999, 110, 9244-9253.

(38) Feng, Y.; Zheng, X. Plasma-enhanced catalytic $\mathrm{CuO}$ nanowires for CO oxidation. Nano Lett. 2010, 10, 4762-4766.

(39) Ren, S.; Bai, Y. F.; Chen, J.; Deng, S. Z.; Xu, N. S.; Wu, Q. B.; Yang, S. Catalyst-free synthesis of $\mathrm{ZnO}$ nanowire arrays on zinc substrate by low temperature thermal oxidation. Mat. Lett. 2007, 61, 666-670.

(40) Hiralal, P.; Unalan, H. E.; Wijayantha, K. G. U.; Kursumovic, A.; Jefferson, D.; MacManus-Driscoll, J. L.; Amaratunga, G. A. J. Growth and process conditions of aligned and patternable films of iron(III) oxide nanowires by thermal oxidation of iron. Nanotechnology 2008, 19, 455608. 


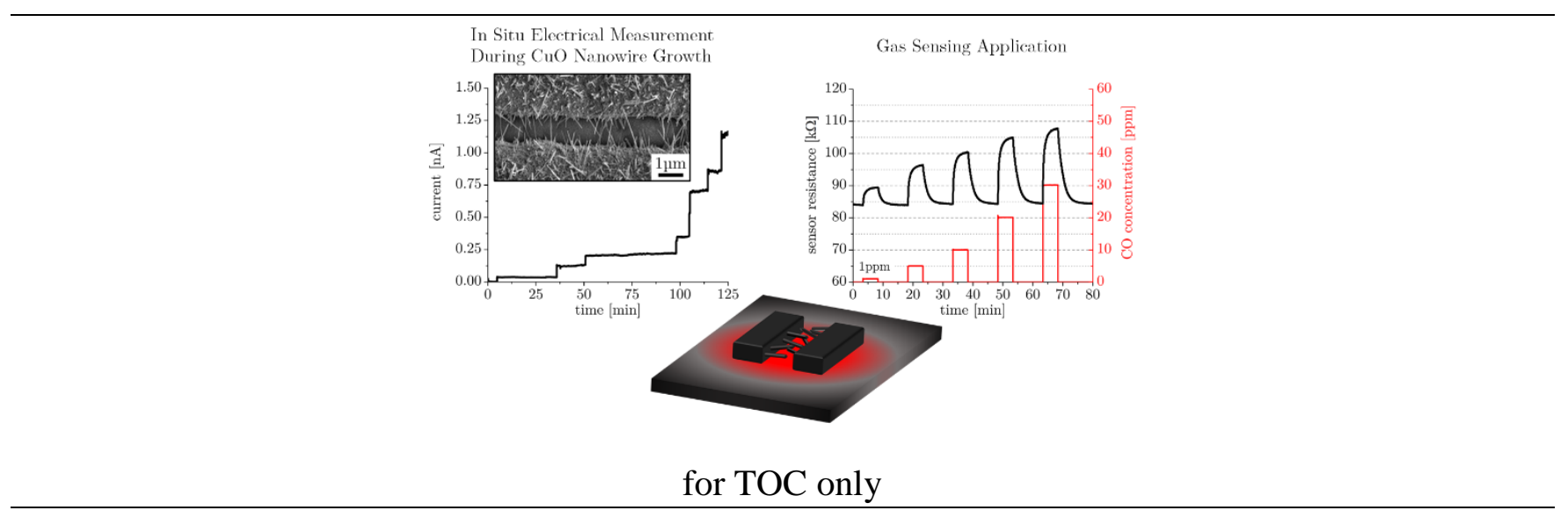

5 